\title{
MONITORAMENTO DA QUALIDADE DE COMPRIMIDOS DE CLONAZEPAM DISTRIBUÍDOS NA REDE PÚBLICA ESTADUAL DE SÃO PAULO E SUA CONTRIBUIÇÃO PARA O SUCESSO TERAPÊUTICO
}

Jaqueline Kalleian Eserian*, Márcia Lombardo

Centro de Medicamentos, Cosméticos e Saneantes, Instituto Adolfo Lutz, São Paulo, SP.

Avenida Doutor Arnaldo, 355, Prédio BQ, $5^{\circ}$ andar, CEP 01246-902, São Paulo, SP.

*E-mail: jkeserian@ial.sp.gov.br

\section{Resumo}

Os benzodiazepínicos são medicamentos tipicamente utilizados para o tratamento de ansiedade e distúrbios do sono, sendo um dos psicotrópicos mais utilizados mundialmente. O clonazepam figura como o medicamento mais vendido no Brasil entre os anos de 2007 e 2010, e está inserido no Programa de Monitoramento da Qualidade de Medicamentos de Saúde Mental do estado de São Paulo. O objetivo deste trabalho foi verificar a qualidade de dez amostras de diferentes lotes de comprimidos de clonazepam 2mg distribuídos em unidades públicas de saúde do estado de São Paulo no período de 2013-2014. Foram realizados ensaios físico-químicos de aspecto; variação de peso; identificação, teor e uniformidade de doses unitárias por cromatografia líquida de alta eficiência. As amostras foram analisadas em um sistema de separação composto de coluna de fase reversa e mistura isocrática ternária, com detecção no ultra-violeta. Os resultados demonstraram que as amostras analisadas estavam satisfatórias quanto aos ensaios realizados. A homogeneidade entre as amostras, conforme observado neste estudo, contribuem, em conjunto com outros fatores, para um tratamento eficaz. Ações de vigilância sanitária que visem ao controle da qualidade de medicamentos distribuídos na rede pública de saúde são imprescindíveis para a garantia de um tratamento farmacológico adequado.

Palavras-chave: Clonazepam, monitoramento, saúde pública, controle de qualidade.

\section{Quality monitoring of Clonazepam tablets distributed through the São Paulo state public network and its contribution to the therapeutic success}

\section{Abstract}

Benzodiazepines drugs are typically used for treating anxiety and sleep disorders and are one of the most prescribed psychotropics worldwide. Clonazepam was the most sold drug in Brazil between 2007 and 2010, and it is part of the drugs chosen for the Mental Health Quality Monitoring Program from the state of São Paulo. The objective of this study was to verify the quality of $2 \mathrm{mg}$ clonazepam tablets from 10 samples with different batches distributed in public health units of the state of São Paulo during 2013-2014. Physical-chemical tests were performed, such as aspect; weight variation; identification, potency and uniformity of dosage units through high performance liquid chromatography. The samples were eluted with ternary isocratic mixture and analyzed by a separation system equipped with reversed-phase column and ultraviolet detector. The results showed that the samples complied with the regulations for the tests carried out. The homogeneity between the samples, as observed in this study, contributes, together with other factors, to an effective treatment. Health surveillance actions aiming the quality control of the medicines distributed through the public health care network are essential to ensure a proper pharmacological treatment.

Keywords: Clonazepam, monitoring, public health, quality control. 


\section{Monitorización de la calidad de comprimidos de Clonazepam distribuidos en la red pública estatal de São Paulo y su contribución al éxito terapéutico}

\section{Resumen}

Los benzodiazepínicos son medicamentos que se usan normalmente para el tratamiento de la ansiedad y los trastornos del sueño, siendo uno de los psicotrópicos más utilizados en todo el mundo. Clonazepam figura como el medicamento más vendido en Brasil entre los años 2007-2010, haciendo parte del Programa de Monitoreo Monitorización de la Calidad de los Medicamentos de Salud Mental del estado de São Paulo. El objetivo de este estudio fue evaluar la calidad de 10 muestras de diferentes lotes de comprimidos de clonazepam 2 mg distribuidos en las unidades públicas de salud del estado de São Paulo en el período 2013-2014. Se Fueron realizados pruebas físico-químicas para examinar la apariencia; variación de peso; identificación, proporción y uniformidad de dosis unitarias por cromatografía de líquida de alta eficiencia. Las muestras fueron analizadas en un sistema de separación compuesto de columna de fase reversa y mezcla isocrática ternaria con detección ultravioleta. Los resultados demostraron que las muestras analizadas estaban satisfactorias con relación a las pruebas realizadas. La homogeneidad de entre las muestras, conforme observado en este estudio, contribuyen, junto con otros factores, para un tratamiento eficaz. Acciones de vigilancia sanitaria dirigidas al control de calidad de los medicamentos distribuidos en la red pública de salud son esenciales para la garantía de un tratamiento farmacológico adecuado.

Palabras clave: Clonazepam, monitorización, salud pública, control de calidad.

\section{INTRODUÇÃO}

Os benzodiazepínicos são medicamentos tipicamente utilizados para o tratamento de ansiedade e distúrbios do sono, sendo um dos psicotrópicos mais utilizados mundialmente, além de serem os mais prescritos para idosos por médicos de família e clínicos gerais ${ }^{(1,2)}$. A prevalência de uso a longo prazo na população geral é de aproximadamente 2-7\% e entre usuários de benzodiazepínicos de $25-76 \%^{(1)}$.

O clonazepam (Figura 1), cujo nome químico é 5-(2-clorofenil)-7-nitro-1,3-diidro-1,4-benzodiazepin-2-ona, é um benzodiazepínico de alta potência que apresenta efeitos anticonvulsivantes, sedativos e ansiolíticos, podendo ser utilizado no tratamento de distúrbios epilépticos; transtornos de ansiedade; transtornos do humor, como adjuvante de estabilizadores de humor na mania aguda e em síndromes psicóticas, como adjuvante na sedação com antipsicóticos na psicose aguda ${ }^{(4)}$. O clonazepam atua de modo seletivo sobre os receptores A do ácido gama-aminobutírico (GABA) potencializando o efeito inibitório deste neurotransmissor ${ }^{(4,5)}$.

Figura 1: Estrutura química do clonazepam.<smiles>O=C1CN=C(c2ccccc2Cl)c2cc([N+](=O)[O-])ccc2N1</smiles>

Fonte: 3. 
De acordo com o último levantamento disponibilizado pela Anvisa sobre o consumo de medicamentos pertencentes à Portaria 344/98, o clonazepam figura como o medicamento mais vendido no Brasil entre os anos de 2007 e $2010^{(6)}$. Em 2007, foram dispensadas 29.463 unidades; em 2008, 4.784.730 unidades; em 2009, 7.498.569 unidades e em 2010, 10.590.047 unidades, ou seja, houve um aumento de mais de $35.000 \%$ no consumo de clonazepam durante este período(6).

Em um levantamento realizado no primeiro semestre de 2013, o Rivotril, que é o medicamento de referência do clonazepam, ficou na $9^{a}$ posição do ranking dos medicamentos mais vendidos no Brasil, sendo prescrito como ansiolítico em cerca de $93 \%$ dos $\operatorname{casos}^{(7)}$.

O Programa de Monitoramento da Qualidade de Medicamentos de Saúde Mental avalia periodicamente a qualidade de medicamentos psicoativos e psicotrópicos distribuídos pela Secretaria de Estado da Saúde (SES) de São Paulo, englobando diversas classes terapêuticas, tais como antidepressivos, ansiolíticos, antipsicóticos e estabilizadores do humor. O clonazepam na forma farmacêutica de comprimidos é um dos medicamentos elencados do programa.

O objetivo deste trabalho foi verificar a qualidade de comprimidos de clonazepam $2 \mathrm{mg}$ distribuídos em unidades públicas de saúde do estado de São Paulo no período de 2013-2014 por meio de análises físico-químicas, com prioridade para os ensaios de aspecto, variação de peso, identificação, teor e uniformidade de doses unitárias. As amostras foram colhidas pela Vigilância Sanitária estadual e os ensaios foram realizados no Instituto Adolfo Lutz (Laboratório Central de Saúde Pública do Estado de São Paulo).

\section{METODOLOGIA}

\subsection{Amostras}

Foram analisadas dez amostras de diferentes lotes de comprimidos de clonazepam de $2 \mathrm{mg}$.

\section{Reagentes e solventes}

Metanol grau HPLC, tetrahidrofurano grau HPLC, fosfato de amônio dibásico anidro e ácido fosfórico foram comprados da Merck (Darmstadt, Alemanha), JT Baker, (Phillipsburg, EUA) e Vetec/Sigma-Aldrich (Duque de Caxias, Brasil), respectivamente. Água ultra-pura 18,2m $\Omega$ foi obtida por um sistema de purificação de água Elgastat Maxima (Veolia, Paris, França). O padrão de referência foi doado pelo Instituto Nacional de Controle de Qualidade em Saúde (INCQS, Rio de Janeiro, Brasil). Filtros com

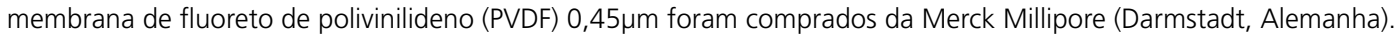

\section{Ensaio de aspecto}

Realizado por inspeção visual dos comprimidos. O produto cumpre o teste se está acondicionado em embalagem lacrada original do fabricante e se os comprimidos não apresentam nenhum tipo de não conformidade, tal como presença de rachaduras, partículas estranhas, esfarelamento e alteração de cor.

\section{Ensaio de variação de peso}

Pesou-se individualmente 20 comprimidos, selecionados aleatoriamente, em balança analítica (Mettler Toledo, AL204, Columbus, USA) e determinou-se o peso médio ${ }^{(8)}$. Tolera-se até duas unidades fora do limite de $\pm 7,5 \%$ em relação ao peso médio, mas nenhuma unidade fora do dobro do limite $( \pm 15 \%)^{(8)}$.

\section{Ensaio de teor e uniformidade de conteúdo de clonazepam em doses unitárias}

Para o ensaio de teor, utilizou-se os comprimidos do ensaio de variação de peso, os quais foram triturados em gral de porcelana até obtenção de um pó fino. A tomada de ensaio foi realizada em duplicata. Para o ensaio de uniformidade de 
conteúdo de clonazepam em doses unitárias, analisou-se dez comprimidos individualmente.

Transferiu-se o pó ou comprimido para balão volumétrico. Para cada balão, adicionou-se aproximadamente $40 \%$ do volume do balão de metanol. Sonicou-se por dez minutos e agitou-se mecanicamente por 20 minutos. Completou-se o volume com diluente, composto de uma mistura de água, metanol e tetrahidrofurano (60:52:13), de modo a se obter uma solução com

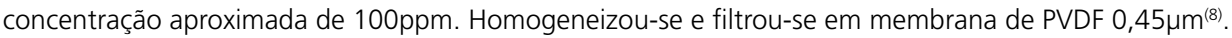

A solução padrão foi preparada nas mesmas condições das amostras por meio da transferência de cerca de $10 \mathrm{mg}$ de clonazepam SQR para balão volumétrico de $100 \mathrm{~mL}$. Adicionou-se aproximadamente $75 \%$ do volume do balão de metanol e sonicou-se por 10 minutos. Completou-se o volume com diluente de modo a se obter uma solução com concentração aproximada de 100ppm. Homogeneizou-se e filtrou-se em membrana de PVDF 0,45 $\mu \mathrm{m}^{(8)}$.

As análises foram realizadas em um cromatógrafo líquido de alta eficiência (HPLC) equipado com um detector UV (Waters, Milford, EUA), utilizando-se colunas analíticas Nucleodur C8 Gravity 250x4.6 mm, 5 mm (Macherey-Nagel, Düren, Alemanha) ou Zorbax C8 250x4.6 mm, 5 um (Agilent, Santa Clara, CA, USA), a $25^{\circ} \mathrm{C}$. A fase móvel consistiu de uma mistura isocrática de tampão fosfato de amônio pH 8,0, metanol e tetrahidrofurano (60:52:13) em um fluxo de $1 \mathrm{~mL} / \mathrm{min}$ e tempo de corrida de 12 minutos. Utilizou-se volume de injeção de $20 \mu \mathrm{L}$ e detecção em $254 \mathrm{~nm}^{(8)}$.

A quantidade de clonazepam foi calculada por comparação da área do pico das soluções amostra com a área do pico da solução padrão, através do software Empower 3.0 (Waters, Milford, EUA). Para o ensaio de teor, o produto cumpre o teste se apresenta de 95 a 110\% da quantidade declarada ${ }^{(8)}$. Para o ensaio de uniformidade de doses unitárias, o produto cumpre o teste se o valor de aceitação (VA) para as dez primeiras unidades testadas é menor que $15 \%$. Se o VA for maior que $15 \%$, testa-se mais 20 unidades. O produto cumpre o teste se o VA para as 30 unidades é menor ou igual a $15 \%$ e nenhuma unidade apresenta conteúdo fora de $75-125 \%$ da quantidade declarada ${ }^{(8)}$.

Ensaio de identificação de clonazepam

Realizado através da comparação do tempo de retenção do pico principal do cromatograma das soluções amostra com àquele da solução padrão(8). O produto cumpre o teste se a identificação é positiva ${ }^{(8)}$.

\section{RESULTADOS E DISCUSSÃO}

Os comprimidos apresentaram aspecto satisfatório, de acordo com os critérios estabelecidos. A variação de peso foi satisfatória, sendo que as amostras apresentaram variação entre $-2,25$ e $+2,85 \%$ (Tabela 1). O ensaio de variação do peso é uma medida indireta do teor do medicamento, uma vez que a fórmula do mesmo contém o princípio ativo diluído em uma mistura de pós homogênea, portanto, espera-se que determinada quantidade de pó apresente quantidade equivalente de princípio ativo(9).

Tabela 1: Resultados dos ensaios de aspecto e variação de peso das amostras analisadas.

\begin{tabular}{llccl}
\hline \multirow{2}{*}{ Amostra } & \multirow{2}{*}{ Aspecto } & \multicolumn{2}{c}{ Variação de peso* } & \multirow{2}{*}{ Resultado } \\
\hline 1 & Satisfatório & $-2,25$ & $+2,85$ & Satisfatório \\
2 & Satisfatório & $-1,39$ & $+1,92$ & Satisfatório \\
3 & Satisfatório & $-1,36$ & $+1,31$ & Satisfatório \\
4 & Satisfatório & $-1,97$ & $+1,96$ & Satisfatório
\end{tabular}




\begin{tabular}{llccl}
\hline \multirow{2}{*}{ Amostra } & \multirow{2}{*}{ Aspecto } & \multicolumn{2}{c}{ Variação de peso } & \multirow{2}{*}{ Resultado } \\
& & $-2,16$ & $+2,40$ & Satisfatório \\
\hline 5 & Satisfatório & $-1,59$ & $+1,36$ & Satisfatório \\
6 & Satisfatório & $-1,20$ & $+1,25$ & Satisfatório \\
7 & Satisfatório & $-1,42$ & $+1,92$ & Satisfatório \\
8 & Satisfatório & $-1,38$ & $+1,61$ & Satisfatório \\
9 & Satisfatório & $-1,55$ & $+1,85$ & Satisfatório \\
\hline 10 & Satisfatório & & \\
\hline
\end{tabular}

*em relação ao peso médio.

A identificação de clonazepam foi positiva em todos os lotes, com um pico correspondente ao padrão de referência em aproximadamente dez minutos (Figura 2). Os lotes apresentaram teor de 97,3 a 102,5\% (Tabela 2). O ensaio de teor permite verificar se a concentração do princípio ativo está de acordo com a quantidade declarada pelo fabricante. A administração de medicamentos com quantidades abaixo ou acima da declarada pode resultar em ineficácia terapêutica ou intoxicação, respectivamente, comprometendo o quadro clínico do paciente ${ }^{(9)}$.

Figura 2: Perfil cromatográfico do comprimido de clonazepam em comparação com o padrão de referência.

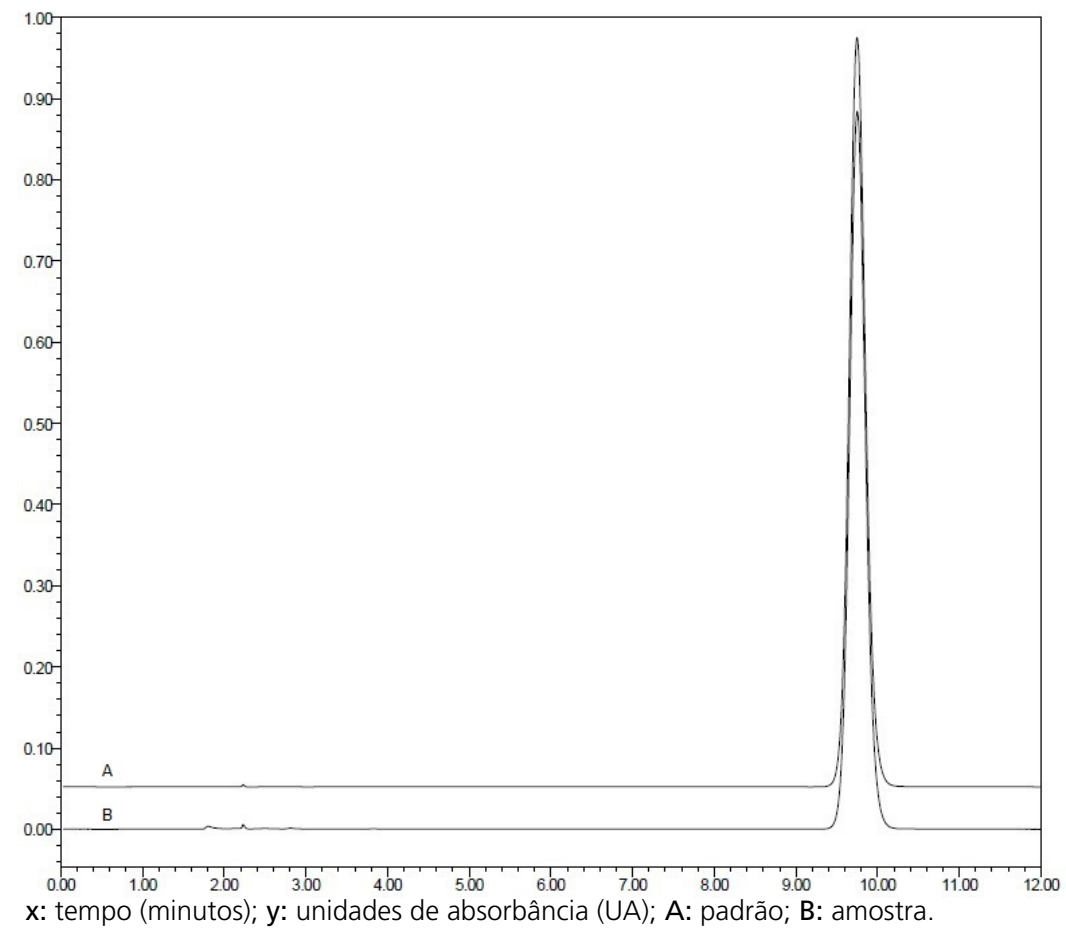


Tabela 2: Resultados dos ensaios de identificação e teor de clonazepam das amostras analisadas.

\begin{tabular}{clll}
\hline Amostra & Identificação & Teor $(\%)$ & Resultado \\
\hline 1 & Positiva & 99,1 & Satisfatório \\
2 & Positiva & 99,3 & Satisfatório \\
3 & Positiva & 102,5 & Satisfatório \\
4 & Positiva & 97,6 & Satisfatório \\
5 & Positiva & 98,4 & Satisfatório \\
6 & Positiva & 99,1 & Satisfatório \\
7 & Positiva & 99,6 & Satisfatório \\
8 & Positiva & 99,4 & Satisfatório \\
9 & Positiva & 97,5 & Satisfatório \\
10 & Positiva & 97,7 & Satisfatório \\
\hline
\end{tabular}

O maior valor de VA observado no ensaio de uniformidade de doses unitárias foi de 5,64\%, evidenciando homogeneidade intra-lote, corroborada pelo desvio padrão relativo, que variou entre 0,71 e 1,70\%. As quantidades mínima e máxima de clonazepam por dose unitária, em porcentagem, foram de 93,25 e 104,38\%, considerando-se todas as amostras (Tabela 3). O ensaio de uniformidade de conteúdo é importante porque permite verificar a quantidade de princípio ativo por comprimido unitariamente, além da homogeneidade de distribuição de princípio ativo entre as unidades representativas do lote.

Tabela 3: Resultados do ensaio de uniformidade de doses unitárias das amostras analisadas.

\begin{tabular}{|c|c|c|c|c|c|}
\hline \multirow[b]{3}{*}{ Amostra } & \multicolumn{4}{|c|}{ Uniformidade de doses unitárias } & \multirow[b]{3}{*}{ Resultado } \\
\hline & \multicolumn{2}{|c|}{ Quantidade de clonazepam } & \multirow{2}{*}{$\begin{array}{l}\text { Valor de } \\
\text { aceitação (\%) }\end{array}$} & \multirow{2}{*}{$\begin{array}{l}\text { Desvio } \\
\text { padrão relativo (\%) }\end{array}$} & \\
\hline & Mínima (\%) & Máxima (\%) & & & \\
\hline 1 & 93,25 & 97,7 & 5,64 & 1,35 & Satisfatório \\
\hline 2 & 95,85 & 100,75 & 4,24 & 1,70 & Satisfatório \\
\hline 3 & 101,37 & 104,38 & 3,74 & 1,08 & Satisfatório \\
\hline 4 & 95,49 & 99,95 & 4,43 & 1,60 & Satisfatório \\
\hline 5 & 95,06 & 99,40 & 3,95 & 1,44 & Satisfatório \\
\hline 6 & 97,18 & 100,52 & 2,63 & 1,11 & Satisfatório \\
\hline 7 & 97,82 & 100,85 & 2,60 & 1,09 & Satisfatório \\
\hline 8 & 94,97 & 100,19 & 3,61 & 1,40 & Satisfatório \\
\hline 9 & 97,11 & 100,87 & 2,87 & 1,21 & Satisfatório \\
\hline 10 & 97,02 & 99,22 & 1,95 & 0,71 & Satisfatório \\
\hline
\end{tabular}


Por ser uma droga potente, a dose de clonazepam administrada deve ser individualizada levando-se em consideração a indicação de uso e características individuais do paciente, tais como resposta clínica, tolerabilidade e idade, a critério médico ${ }^{(4)}$.

Doses menores administradas em intervalos maiores são requeridas para pacientes idosos, devido à eliminação plasmática mais lenta. Além disso, efeitos adversos de benzodiazepínicos são mais comuns em pacientes idosos, ocorrendo com maior frequência com o avanço da idade ${ }^{(10)}$. Doses que têm efeito terapêutico para pacientes entre 65-70 anos podem produzir efeitos adversos significativos em pacientes com idade maior que 75 anos ${ }^{(10)}$, portanto, a prescrição deve ser cautelosa e, de preferência, por um período curto(2).

Variações na quantidade de clonazepam intra e inter-lotes podem acarretar em efeitos clínicos significativos. A homogeneidade entre as amostras com relação ao teor e ao conteúdo de clonazepam por dose unitária, conforme observado neste estudo, contribuem, em conjunto com outros fatores, para um tratamento eficaz.

De acordo com a Lei 9782/99(11), a Agência Nacional de Vigilância Sanitária (ANVISA) tem por objetivo a promoção da proteção da saúde da população por meio de ações de controle sanitário tanto da produção quanto da comercialização de produtos e serviços submetidos à vigilância sanitária, tais como medicamentos.

Uma das importantes ações da Vigilância Sanitária é o monitoramento da qualidade de medicamentos ${ }^{(9)}$. A administração de medicamentos de qualidade, seguros e eficazes é fundamental, uma vez que é parte significativa da melhoria ou cura de processos patológicos(9).

A distribuição de medicamentos de qualidade na rede pública está diretamente relacionada à atenção da saúde, contribuindo para a resolução das ações nesta área. $O$ crescente aumento dos custos na área da saúde fazem com que a gestão de medicamentos seja um ponto crítico na administração pública. Além dos custos referentes à aquisição de medicamentos em grande quantidade, a qualidade do produto adquirido tem grande impacto econômico no sistema público de saúde ${ }^{(12)}$. Medicamentos com desvio de qualidade podem gerar risco de vida ao paciente, vindo a onerar ainda mais o sistema público de saúde, gerando novas consultas médicas no Sistema Único de Saúde (SUS), solicitação de novos exames e dispensação de outros medicamentos ${ }^{(12)}$, além da interdição e recolhimento de lotes.

Os programas de monitoramento da qualidade de medicamentos distribuídos na rede pública têm o objetivo de verificar periodicamente a qualidade de determinados medicamentos elencados de acordo com o interesse para a saúde pública. Este tipo de ação é uma forma de intervenção preventiva, na qual os produtos são analisados antes de qualquer dano ou agravo em potencial(13). Os resultados obtidos a partir destes programas atuam como indicadores em vigilância sanitária, possibilitando a tomada de ações estratégicas.

\section{CONCLUSÃO}

Ações de vigilância sanitária que visem ao controle da qualidade de medicamentos distribuídos na rede pública de saúde são imprescindíveis para a garantia de um tratamento farmacológico adequado. Os resultados demonstraram que as amostras analisadas estavam satisfatórias quanto aos ensaios realizados. O controle periódico da qualidade de comprimidos de clonazepam se faz necessária, uma vez que a falta de homogeneidade entre os comprimidos pode vir a comprometer o tratamento clínico. 


\section{REFERÊNCIAS}

1. Quaglio G, Pattaro C, Gerra G, Mathewson S, Verbanck P, Des Jarlais DC et al. High dose benzodiazepine dependence: description of 29 patients treated with flumazenil infusion and stabilised with clonazepam. Psychiatry Res.

2012;198(3):457-462.

2. Sithamparanathan K, Sadera A, Leung L. Adverse effects of benzodiazepine use in elderly people: a meta-analysis. Asian J. Gerontol. Geriatr. 2012;7(2):107-11.

3. The US Pharmacopoeia. 38th. ed. Rockville: The US Pharmacopoeial Convention, 2015.

4. Cordioli A. Psicofármacos: consulta rápida. $2^{\mathrm{a}}$ ed. Porto Alegre: Artmed; 2000.

5. Rang HP, Dale MM, Ritter JM, Moore PK. Farmacologia. 5a ed. Rio de Janeiro: Elsevier; 2005.

6. Agência Nacional de Vigilância Sanitária [Internet]. Brasília: Ministério da Saúde (BR) [cited 2015 jun 11]. Boletim de Farmacoepidemiologia SNGPC, v.2, ano 1, 2011. Available from: http://www.anvisa.gov.br/sngpc/boletins/2011/boletim_ sngpc_2edatualizada.pdf

7. Agência Nacional de Vigilância Sanitária [Internet]. Brasília: Ministério da Saúde (BR) [cited 2015 jun 11]. Saúde e economia, n 10, ano V, 2013. Available from: http://portal.anvisa.gov.br/wps/wcm/connect/e2b81e804680dc1186939699223cd76e/ saude_economia\%2B10\%2B2013.pdf?MOD=AJPERES

8. Farmacopeia Brasileira. 5. ed. v.2, Brasília: Anvisa, 2010.

9. Peixoto MM, Júnior AFS, Santos CAA, Júnior EC. Avaliação da qualidade de comprimidos de captopril dispensados em Feira de Santana - BA. Infarma. 2005;16(13-14):69-73.

10. Bogunovic OJ, Greenfield SF. Use of benzodiazepines among elderly patients. Psychiatr. Serv. 2004;55(3):233-235.

11. Lei N. 9.782 de 26 de janeiro de 1999. Define o Sistema Nacional de Vigilância Sanitária, cria a Agência Nacional de Vigilância Sanitária, e dá outras providências. Diário Oficial da União (Brasília). 1999 Jan 27.

12. Bianchin MD, Blatt CR, Soares AS, Külkamp-Guerreiro IC. Avaliação da qualidade de comprimidos de propranolole enalapril distribuidos no sistema público de saúde em uma cidade do sul do Brasil. Ciênc. saúde colet. 2012;17(2):491498.

13. Pontes Junior DM, Pepe VLE, Osorio-de-Castro CGS, Massena EP, Portela MC, Miranda MC et al. A definição de medicamentos prioritários para o monitoramento da qualidade laboratorial no Brasil: articulação entre a vigilância sanitária e a Política Nacional de Medicamentos. Cad. Saúde Pública. 2008;24(9):2081-2090. 